\title{
Enantio-, regio-, and chemoselective reduction of aromatic a- diketones by baker's yeast in diverse organic-water solvent systems
}

\author{
N. O. Mahmoodi* and M. Noori Navrood \\ Organic Research Laboratory, Department of Chemistry, University of Guilan, \\ Rasht, P.O. Box 1914, Iran \\ E-mail: mahmoodi@guilan.ac.ir
}

Dedicated to Prof. Ernst Anders on the occasion of his 65th anniversary

\begin{abstract}
The enantio- and regioselective reduction of several symmetric and nonsymmetrical parasubstituted benzil derivatives was achieved utilizing Saccharomyces cerevisiae (baker's yeast) in solvents such as EtOAc, $\mathrm{Et}_{2} \mathrm{O}, n$-hexane, $n$-pentane and toluene in the presence of $14-33 \%$ of water. For compounds (1c) and (3b) using organic solvents gave significantly better yields. However, (1a), (1b) and (3a) give comparable yields, regardless of solvent. In most cases the reduction in organic-water solvent systems gave higher ee.
\end{abstract}

Keywords: Organic-water solvent systems, chemoselective, Saccharomyces cerevisiae, asymmetric synthesis, baker's yeast

\section{Introduction}

Biocatalysis is one of the most important stereoselective preparations of optically active compounds. ${ }^{1}$ Baker's yeast (Saccharomyces cerevisiae) mediated enzymatic transformations of organic compounds are well known reactions in organic chemistry. The reduction of benzils (1) and (3) using Baker's yeast is highly desirable. Chiral $\alpha$-keto alcohols are important intermediates for the production of pharmaceuticals, flavors and fragrances. ${ }^{2}$ They are also remarkable synthons for the asymmetric synthesis of natural products. ${ }^{3,4}$ Although there are few articles on the enantioselective reduction of $\alpha$-diketones ${ }^{5 \mathrm{a}-5 \mathrm{c}, 5 \mathrm{~g}}$ but the $\beta$-ketoesters reduction with Baker's yeast (Saccharomyces cerevisiae) has been widely studied. Recently we reported the enantioselective reduction of $\beta$-ketoesters, ${ }^{5 \mathrm{f}}$ enantio-, region-, and chemoselective reduction of several symmetric and nonsymmetrically para-substituted benzil derivatives ${ }^{5 a}$ and enantioselective reduction of $\gamma$-ketoacids and related $\gamma$-ketoesters and their direct conversion to the corresponding chiral 5-aryl lactones in the presence of Saccharomyces cerevisiae. ${ }^{5 \mathrm{e}}$ Yeasts 
are economical catalysts, are neither toxic nor pathogenic and the reaction can be carried out at moderate reaction conditions. ${ }^{6,7}$ Many $\beta$-ketoesters and $\alpha$-diketones show low water solubility and are toxic for yeast cells. These results in low reaction rates and yields in water medium, furthermore the observed enantiomeric excess is often low due to the competing activities of many oxidoreductases in yeast cells.

Replacing the more customary aqueous reaction environment, an organic solvent has been used as a medium for yeast reactions. ${ }^{7-11}$ The main advantage associated with the use of an organic solvent is the simplicity with which pure product can be isolated and has been shown that significantly better yields and enantioselectivities can be achieved. ${ }^{12}$

\section{Results and Discussion}

We now present the reduction of $\alpha$-diketones in several organic solvents such as EtOAc, $\mathrm{Et}_{2} \mathrm{O}, n$ hexane, toluene and $n$-pentane containing 14-33 \% v/v water (Scheme 1). Our results (Table I) indicate that the enantioselectivity and isolated yields obtained in organic-water solvents in many cases are superior to that achieved in the corresponding aqueous medium. From the standpoint of asymmetric diaryl synthesis similar to the water medium, the reduction encompasses three problems: chemoselectivity, regioselectivity, and enantioselectivity. ${ }^{5 \mathrm{a}}$

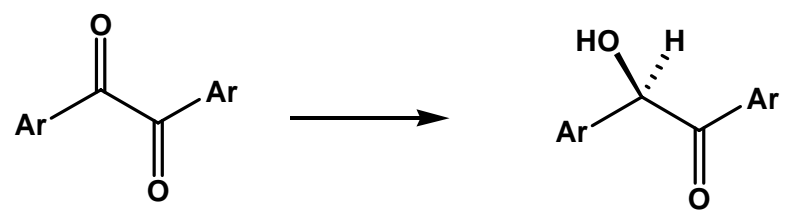

1a) $\mathrm{Ar}=\mathrm{Ph}$

1b) $\mathrm{Ar}=$ furyl

1c) $\mathrm{Ar}=4-\mathrm{Me}-\mathrm{C}_{6} \mathrm{H}_{4}-$
(R)-2a $\mathrm{Ar}=\mathrm{Ph}$

(R)-2b Ar = furyl

(R)-2c Ar $=4-\mathrm{Me}^{-} \mathrm{C}_{6} \mathrm{H}_{4}-$

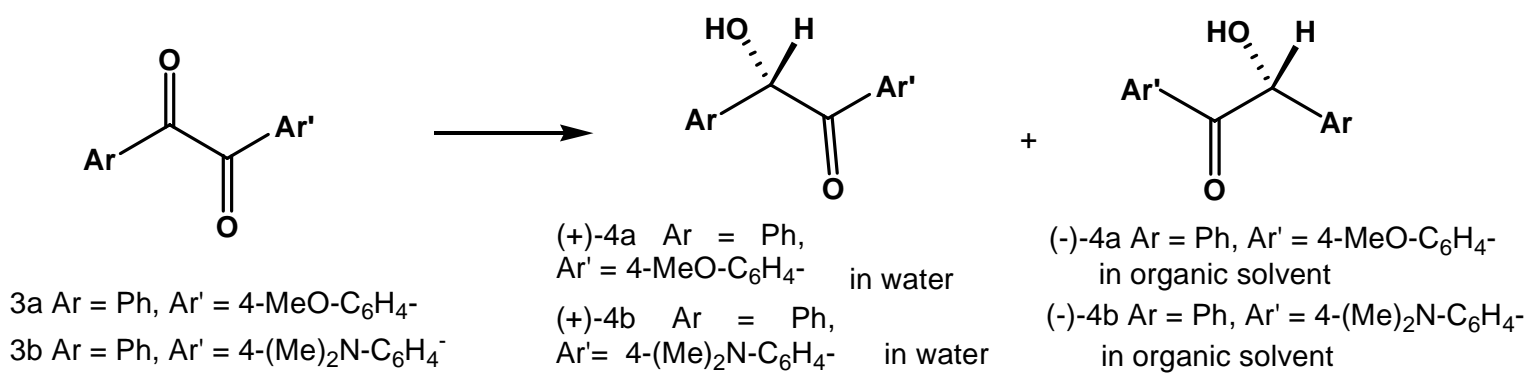

\section{Scheme1}

The main advantage associated with the use of an organic solvent is the simplicity of workup procedure and we have shown that better yields and enantioselectivities as well as regioselectivity and chemoselectivity for (3a) and (3b) were obtained (Tables 1). However, from point of enantioselectivity in contrast to the water medium for (4a) and (4b) conversion of 
stereochemistry was observed (Scheme 1). The use of series of pre-made $\alpha$-diketones ${ }^{13}$ (1a), (1b), (1c), (3a) and (3b) for examining yeast mediated reduction in organic solvents was achieved. TLC examination indicates that deactivation of the reductase enzymes began after about $10-12 \mathrm{~h}$ in most of the organic solvent systems and that after $24 \mathrm{~h}$ slight activity remained. For this reason in many reduction systems dry active yeast and $(+)$-D-glucose was added to the reaction flask several times and after every $\sim 10 \mathrm{~h}$ periods. The deactivation was highly temperature-dependent; at $30-33{ }^{\circ} \mathrm{C}$ enzymatic activity had finished after about $10 \mathrm{~h}$ while at 10 $15{ }^{\circ} \mathrm{C}$ no decrease of enzymatic activity could be observed, even after $65-75 \mathrm{~h}$. The chemo, and regioselectivity in organic solvents in addition to water medium was confirmed by mean of ${ }^{1} \mathrm{HNMR}$ spectra in $\mathrm{CD}_{3} \mathrm{COCD}_{3}$ of (-)-4a and (-)-4b after shaking the NMR tube with $\mathrm{D}_{2} \mathrm{O}$. The doublet peaks at 4.5 and $4.7 \mathrm{ppm}$ due to the $\alpha$-hydrogen $(\mathrm{C}-\mathrm{H})$ adjacent to the unsubstituted phenyl rings in both (-)-4a and (-)-4b spectra removed. ${ }^{13} \mathrm{CNMR}$ spectra were run on a Bruker DRX500 spectrometer operating at $125 \mathrm{MHz}$. The dried baker's yeast $0.1 \mathrm{~g}$ mediated reduction of (3a) $(0.1 \mathrm{mmol})$ in hexane $3 \mathrm{ml}, \mathrm{CD}_{3} \mathrm{COCD}_{3}$ a lock substance, and water $80 \mu \mathrm{l}$ was examined in an ${ }^{13} \mathrm{CNMR}$ tube at room temperature $\sim 20^{\circ} \mathrm{C}$ and a ${ }^{13} \mathrm{CNMR}$ spectrum recorded. With regard to the two carbonyl peaks of (3a) at 192.76 for C2 and 194.55 for $\mathrm{C} 1$, the peak at $192.76 \mathrm{ppm}$, due to $\mathrm{C} 2$ of carbonyl group of (3a), disappeared and the peak at $75.16 \mathrm{ppm}$, due to $\mathrm{C} 2$ of the reduced product (4a), appeared. The comparison time for appearances and disappearances of the C2-3a and C2-4a peaks varied from experiment to experiment (Scheme 2).

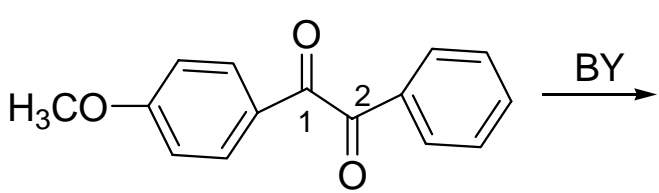

$3 a$

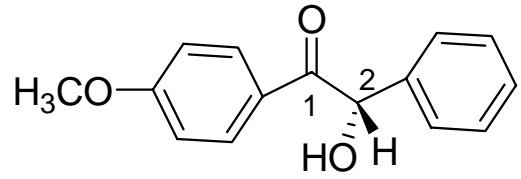

$(-)-4 a$

\section{Scheme 2}

The proportion of these peaks clearly shows the formation of the product and the consumption of the starting material. It was determined that in most of organic solvents especially $n$-hexane the enzyme activity remained constant for about $10 \mathrm{~h}$ and then rapidly decreased until after $24 \mathrm{~h}$ insufficient activity remained. Selectivity of yeast reductions are not always satisfactory, different methods have been developed to improve the stereochemical result $^{14}$. 
Table 1. Preparative conversions with Saccharomyces cervisia in aqueous fermenting' medium

\begin{tabular}{|c|c|c|c|c|c|c|c|c|}
\hline Solvent & Entry & $(R)-\mathbf{2 a}$ & $(R)-\mathbf{2 b}$ & $(R)-2 \mathrm{c}$ & $(+)-4 a$ & $(-)-4 a$ & $(+)-4 b$ & $(-)-4 b$ \\
\hline \multirow{3}{*}{ EtOAc } & Yield \% & 44.3 & 33 & 60 & & 50.7 & - & 51.43 \\
\hline & ee $\%$ & 60 & 95.9 & 54.2 & - & & - & \\
\hline & {$[\mathrm{a}]_{\mathrm{D}}{ }^{25}$} & $\begin{array}{c}-69 \\
\text { Et2O }\end{array}$ & $\begin{array}{l}-19.3 \\
\text { acetone }\end{array}$ & $\begin{array}{l}-78.3 \\
\text { acetone }\end{array}$ & - & $\begin{array}{c}-84 \\
\text { acetone }\end{array}$ & - & $\begin{array}{l}-56.4 \\
\text { acetone }\end{array}$ \\
\hline \multirow{3}{*}{$(\mathrm{Et}) 2 \mathrm{O}$} & Yield \% & 50 & 55 & 56 & - & 41.3 & - & 42.86 \\
\hline & ee $\%$ & 46.9 & 71.8 & 60.2 & - & & - & - \\
\hline & {$[\mathrm{a}]_{\mathrm{D}}{ }^{25}$} & $\begin{array}{c}-54 \\
\text { Et2O }\end{array}$ & $\begin{array}{l}-14.5 \\
\text { acetone }\end{array}$ & $\begin{array}{l}-88.6 \\
\text { acetone }\end{array}$ & - & $\begin{array}{l}-20.8 \\
\text { acetone }\end{array}$ & - & $\begin{array}{l}-42.6 \\
\text { acetone }\end{array}$ \\
\hline \multirow{3}{*}{ n-hexane } & Yield \% & 54.3 & 46.6 & 47 & - & 48 & - & 65.7 \\
\hline & ee $\%$ & 85.5 & 83.5 & 50.8 & - & - & - & - \\
\hline & {$[\mathrm{a}]_{\mathrm{D}}{ }^{25}$} & $\begin{array}{l}-98.5 \\
\text { Et2O }\end{array}$ & $\begin{array}{l}-16.8 \\
\text { acetone }\end{array}$ & $\begin{array}{l}-74.8 \\
\text { acetone }\end{array}$ & - & $\begin{array}{l}-86.3 \\
\text { acetone }\end{array}$ & - & $\begin{array}{l}-79.4 \\
\text { acetone }\end{array}$ \\
\hline \multirow{3}{*}{ toluene } & Yield \% & 42.8 & 51.7 & 62 & - & 54.7 & - & 60 \\
\hline & $\mathrm{ee} \%$ & 76 & 58.6 & 61 & - & - & - & - \\
\hline & {$[\mathrm{a}]_{\mathrm{D}}{ }^{25}$} & $\begin{array}{l}-85.5 \\
\text { Et2O }\end{array}$ & $\begin{array}{l}-11.8 \\
\text { acetone }\end{array}$ & $\begin{array}{l}-89.8 \\
\text { acetone }\end{array}$ & - & $\begin{array}{l}-43.8 \\
\text { acetone }\end{array}$ & - & $\begin{array}{c}-38 \\
\text { acetone }\end{array}$ \\
\hline \multirow{3}{*}{ n-pentane } & Yield \% & 41.4 & - & - & - & - & - & - \\
\hline & ee $\%$ & 95.6 & - & - & - & - & - & - \\
\hline & {$[\mathrm{a}]_{\mathrm{D}}{ }^{25}$} & $\begin{array}{l}-110 \\
\text { Et2O }\end{array}$ & - & - & - & - & - & - \\
\hline \multirow{3}{*}{ water } & Yield \% & 43 & 41 & 17 & 47 & - & 21 & - \\
\hline & $\mathrm{ee} \%$ & 50 & 82 & 36 & - & - & & - \\
\hline & $\begin{array}{l}{[\mathrm{a}]_{\mathrm{D}}{ }^{25}} \\
\mathrm{Et} 2 \mathrm{O}\end{array}$ & $\begin{array}{l}-57.5 \\
\text { Et2O }\end{array}$ & $\begin{array}{l}-16.5 \\
\text { acetone }\end{array}$ & $\begin{array}{c}-53 \\
\text { acetone }\end{array}$ & $\begin{array}{c}+70 \\
\text { acetone }\end{array}$ & - & +47 & - \\
\hline
\end{tabular}

In conclusion, we have shown that, yeast was successfully employed in the asymmetric hydrogenations of prochiral $\alpha$-diketones to the corresponding $\alpha$-hydroxy ketones in organic solvent/water mixtures; and increased enantioselectivity (up to 97\% ee) and yields, in addition for facilitated isolation, were observed using these conditions. The enantioselectivity and isolated yields obtained in organic/water mixtures are somewhat higher for compounds (1c) and (3b). However dibenzils (1a), (1b) and (3a) give comparable yields, regardless of solvent and higher to that achieved in the corresponding aqueous medium. The ${ }^{13} \mathrm{CNMR}$ experiments and TLC monitoring indicated that the deactivation of the reductase enzymes began after about $10 \mathrm{~h}$ in the organic solvent systems and that after $24 \mathrm{~h}$ little activity remained. The yield of conversion and highest ee $\%$ for $(R)$-2a was obtained in $n$-hexane $(54.3 \%$, ee $85.6 \%)$ and $n$-pentane $(41.5 \%$, ee $95.6 \%)$ which compares with water $(43 \%$, ee, $50 \%)$. For $(R)-2 b$ in $\mathrm{Et}_{2} \mathrm{O}(55 \%$, ee $71.8 \%)$, in 
EtOAc $(35 \%$, ee $95.9 \%)$ in water (41\%, ee $82 \%)$; for $(R)-2 \mathrm{c}$ in toluene $(62 \%$, ee $61 \%)$, in water $(17 \%$, ee $36 \%)$ was obtained respectively. Reduction of (3a) in toluene gave $(54.7 \%)$ of (-) - $4 \mathbf{a}$, while reduction in water provides opposite stereochemistry $(+)-(\mathbf{4 a})$ with $47 \%$ yield; for (3b) reduction in $n$-hexane gave $(65.7 \%)$ of $(-)-4 \mathbf{b}$, while reduction in water offered opposite stereochemistry $(+)-\mathbf{4 b}(21 \%)$ (Table 1$)$.

\section{Experimental Section}

General Procedures. Melting points are uncorrected and determined by Mettler Fp5 melting point apparatus. IR spectra were obtained on a Shimadzu IR-470. Products were characterized by IR, NMR, GC-MS, TLC, and mp. All NMR data were recorded in $\mathrm{CDCl}_{3}$ or $\mathrm{CD}_{3} \mathrm{COCD}_{3}$ using a Bruker Avance $500-\mathrm{MHz}$ spectrometer. Chemical shifts are reported in ppm $(\delta)$ using TMS as internal reference. Mass spectra were obtained from a GC-MS Agilent Technologies QP-5973N MSD instrument.

Chemicals were purchased from Fluka, Merck, and Aldrich. Commercial baker's yeast, natural yeast, S. cerevisiae as active and dry material (Saf-levure, S.I. Lesaffre 59703, Mareq, France) was used. Yields refer to isolated pure center cut from column chromatography or for material scratched from preparative TLC plates. The specific rotation, $[\mathrm{a}]_{\mathrm{D}}$, was determined on a commercial polarimeter ATAGO (POLAX) (cell path lengths of $10 \mathrm{~cm}$ were used). The UV-Vis spectra were recorded on a Shimadzu UV-2100. Compounds (Ia)-(Ic) were prepared from their respective $\alpha$-hydroxy ketones (3a), and (3b) by oxidation with $\mathrm{NH}_{4} \mathrm{NO}_{3}$, in the presence of $\mathrm{Cu}\left(\mathrm{CH}_{3} \mathrm{COO}\right)_{2}$ in acetic acid according to ref. ${ }^{15}$, for the oxidation of (3b) $\mathrm{CuSO}_{4}$ and pyridine were utilized ${ }^{16}$. These $\alpha$-hydroxy ketone adducts were obtained by benzoin condensation ${ }^{17}$.

Reduction of (1a) in presence of baker's yeast in n-pentane. Preparation of $(R)$-2-hydroxy1,2-diphenylethanone ((R)-2a, $\left.\mathrm{C}_{14} \mathrm{H}_{12} \mathrm{O}_{2}\right)$ : a typical procedure

To a $250 \mathrm{~mL}$ round bottom flask was added $5 \mathrm{~g}$ of $\mathrm{D}-(+)$-glucose monohydrate $(25.2 \mathrm{mmol}), 3$ $\mathrm{mL}$ of distilled $\mathrm{H}_{2} \mathrm{O}$ and $3 \mathrm{~mL}$ of potassium dihydrogen phosphate buffer $(\mathrm{pH}=7,0.1 \mathrm{M})$. The resulting mixture was stirred at room temperature for several minutes to produce a homogeneous solution whereupon $5 \mathrm{~g}$ of active dry yeast were added. The solution was stirred for $30 \mathrm{~min}$ at $30-40{ }^{\circ} \mathrm{C}$. The flask was equipped with a bent glass tube that through a trap dips below the surface of a saturated aqueous solution of $\mathrm{Ba}(\mathrm{OH})_{2}$ in a $200 \mathrm{~mL}$ Erlenmeyer flask. After activation of yeast it happening with evolving carbon dioxide, $0.7 \mathrm{~g}$ (3.3 mmol) of (1a) was dissolved in $25 \mathrm{~mL} n$-pentane and were added dropwise to the reaction mixture during $30 \mathrm{~min}$. After $10 \mathrm{~h}, 5 \mathrm{~g}$ of active dry yeast and $5 \mathrm{~g} \mathrm{D}-(+)$-glucose monohydrate $(25.2 \mathrm{mmol})$ with $2 \mathrm{~mL}$ distilled water were added. The solution was stirred vigorously at $30^{\circ} \mathrm{C}$. After $24 \mathrm{~h}$, the solution was extracted three times with $25 \mathrm{~mL}$ portions of $n$-pentane. The combined organic layers were dried $\left(\mathrm{MgSO}_{4}\right)$ and the solvent was evaporated under reduced pressure to leave the crude product as a residue. The crude product was purified on a silica gel column. The elution solvents was 1:3 $(\mathrm{v}: \mathrm{v})$ of $\mathrm{Et}_{2} \mathrm{O}$ :ligroin to afford $0.29 \mathrm{~g}$ of $(R)-2 \mathrm{a}(41.4 \%), \mathrm{mp} 135^{\circ} \mathrm{C},[\alpha]_{\mathrm{D}}{ }^{25}=-110(\mathrm{c}=0.047 \mathrm{M}$, 
$\left.\mathrm{Et}_{2} \mathrm{O}\right)$, ee $=95.6 \%\left(\right.$ commercially available $\mathrm{S}$ product, $\left.\mathrm{CH}_{3} \mathrm{COCH}_{3}, \mathrm{c}=1.5,[\mathrm{a}]_{\mathrm{D}}{ }^{25}=+115\right), \mathrm{mp}$ 134-136 ${ }^{\circ} \mathrm{C}$. IR (KBr):3400 (OH), 3070 (C-H stretching), $1680(\mathrm{C}=\mathrm{O}), 1580,1510,1440,1205$, 1165, 875, 740, $720 \mathrm{~cm}^{-1} ;{ }^{1} \mathrm{H}$ NMR $\left(\mathrm{CDCl}_{3}\right), \delta: 7.9(\mathrm{dd}, \mathrm{J}=6.8 \mathrm{~Hz}, 2 \mathrm{H}, \mathrm{ArH}), 7.4(\mathrm{~m}, 8 \mathrm{H}, \mathrm{ArH})$, $5.9(\mathrm{~s}, 1 \mathrm{H}, \mathrm{CH}), 4.5($ broad s, $1 \mathrm{H}, \mathrm{OH}) \mathrm{ppm} ;{ }^{13} \mathrm{C} \mathrm{NMR}\left(\mathrm{CDCl}_{3}\right), \delta: 76.50(\mathrm{CH}), 128.00(\mathrm{C})$, 129.10 (C), 129.30 (C), 129.79 (C), 133.16 (C), 134.26 (C), 139.06 (C), 199.10 (C=O) ppm.

Reduction of 1,2-di(furan-2-yl)ethane-1,2-dione (1b) by baker's yeast in $\mathrm{Et}_{2} \mathrm{O}$ : (R)-2hydroxy-1-(4-methoxyphenyl)-2-phenyl-ethanone $\left((R)-2 b, \mathrm{C}_{15} \mathrm{H}_{14} \mathrm{O}_{3}\right)$ : a typical procedure

To a $250 \mathrm{~mL}$ round bottom flask was added $5 \mathrm{~g}$ of D-(+)-glucose monohydrate $(25.2 \mathrm{mmol}), 3 \mathrm{~mL}$ of distilled $\mathrm{H}_{2} \mathrm{O}$ and $3 \mathrm{~mL}$ of potassium dihydrogen phosphate buffer $(\mathrm{pH}=7,0.1 \mathrm{M})$. The resulting mixture was stirred at room temperature for several minutes to produce a homogeneous solution whereupon $5 \mathrm{~g}$ of active dry yeast were added. The solution was stirred for $30 \mathrm{~min}$ at $30-40{ }^{\circ} \mathrm{C}$. The flask was equipped with a bent glass tube that through a trap dips below the surface of a saturated aqueous solution of $\mathrm{Ba}(\mathrm{OH})_{2}$ in a $200 \mathrm{~mL}$ Erlenmeyer flask. After activation of yeast it happening with evolving carbon dioxide, $0.6 \mathrm{~g}(2.85 \mathrm{mmol})$ of (1b) was dissolved in $25 \mathrm{~mL} \mathrm{Et}_{2} \mathrm{O}$ and were added dropwise to the reaction mixture during $30 \mathrm{~min}$. After $24 \mathrm{~h}, 3 \mathrm{~g}$ of active dry yeast and $3 \mathrm{~g} \mathrm{D}-(+)$-glucose monohydrate $(15.12 \mathrm{mmol})$ with $5 \mathrm{~mL} \mathrm{Et}_{2} \mathrm{O}$ were added. The solution was stirred vigorously at $30^{\circ} \mathrm{C}$. After $44 \mathrm{~h}$ the solution was extracted three times with $25 \mathrm{~mL}$ portions of $\mathrm{Et}_{2} \mathrm{O}$. The combined organic layers were dried $\mathrm{MgSO}_{4}$ and the solvent was evaporated under reduced pressure to leave the crude product as a residue. The crude product was purified on a silica gel column. The elution solvent was 1:3 (v:v) of $\mathrm{Et}_{2} \mathrm{O}$ :ligroin to afford $0.33 \mathrm{~g}$ of $(R)-2 \mathbf{b}(55 \%), \mathrm{mp} 138^{\circ} \mathrm{C},[\mathrm{a}]_{\mathrm{D}}{ }^{25}=-14.45(\mathrm{c}=0.052 \mathrm{M}$, acetone), ee $=71.8 \%$ (lit. for commercially available $(S)-2 \mathbf{b}, \mathrm{Et}_{2} \mathrm{O}, \mathrm{C}=2 \mathrm{M},[\mathrm{a}]_{\mathrm{D}}{ }^{25}=+20.1$, mp 134-137 ${ }^{\circ} \mathrm{C}^{17}$. IR (KBr): $3400(\mathrm{OH}), 3100$ (C-H stretching), 2940 (C-H stretching), $1665(\mathrm{C}=\mathrm{O}), 1460$, 1250, 1145, $1035,910,780,735 \mathrm{~cm}^{-1} ;{ }^{1} \mathrm{H} \mathrm{NMR}\left(\mathrm{CDCl}_{3}\right), \delta: 4.2(\mathrm{~d}, \mathrm{~J}=6.6 \mathrm{~Hz}, 1 \mathrm{H}, \mathrm{OH}), 5.8(\mathrm{~d}$, $\mathrm{J}=6.6 \mathrm{~Hz}, 1 \mathrm{H}, \mathrm{CH}), 6.4(\mathrm{dd}, 2 \mathrm{H}, \mathrm{J}=3 \mathrm{~Hz}, \operatorname{ArH}), 6.6(\mathrm{dd}, 1 \mathrm{H}, \mathrm{J}=3.1 \mathrm{~Hz}, \operatorname{ArH}), 7.3(\mathrm{dd}, 1 \mathrm{H}, \mathrm{J}=$ $1 \mathrm{~Hz}, \operatorname{ArH}), 7.4(\mathrm{dd}, 1 \mathrm{H}, \mathrm{J}=1 \mathrm{~Hz}, \mathrm{ArH}), 7.7(\mathrm{dd}, \mathrm{J}=1 \mathrm{~Hz}, 1 \mathrm{H}, \mathrm{Ar} \mathrm{H}) \mathrm{ppm} ;{ }^{13} \mathrm{C} \mathrm{NMR}\left(\mathrm{CDCl}_{3}\right)$ $\delta$ : 69.10 (CH), 109.21 (CAr), 111.08 (C), 113.16 (C), 120.37 (C), 144.54 (C), 148.21 (C), 158.09 (C), $167.27(\mathrm{C}), 184.50(\mathrm{C}=\mathrm{O}) \mathrm{ppm}$.

Reduction of 4,4'-dimethyl benzyl (Ic) by baker's yeast in EtOAc : (R)-2-hydroxy-1,2-di(ptolyl) ethanone ((R)-2c $\left.\mathrm{C}_{16} \mathrm{H}_{16} \mathrm{O}_{2}\right)$

A similar procedure as used for (1a) was applied. The elution solvent was 1:3 (v:v) $\mathrm{Et}_{2} \mathrm{O}$ :ligroin to afford $0.3 \mathrm{~g}, 60 \%$ of $(R)-2 \mathrm{c}, \mathrm{mp} 87^{\circ} \mathrm{C}$, (acetone, $\mathrm{c}=0.042 \mathrm{M},[\mathrm{a}]_{\mathrm{D}}{ }^{25}=-78.3$ (commercially available product $[\mathrm{a}]_{\mathrm{D}}{ }^{25}-130.8(\mathrm{c}=1, \mathrm{MeOH}), \mathrm{mp} 89{ }^{\circ} \mathrm{C}{ }^{17}$. IR $(\mathrm{KBr}): 3450(\mathrm{OH}), 3020(\mathrm{CH}$ stretching), 2910 (CH stretching), $1675(\mathrm{C}=\mathrm{O}), 1500,1510,1390,1100,970,850,820740,780$ $\mathrm{cm}^{-1} ;{ }^{1} \mathrm{H} \mathrm{NMR}\left(\mathrm{CDCl}_{3}\right) \delta: 2.3\left(\mathrm{~s}, 3 \mathrm{H}, \mathrm{CH}_{3}\right), 2.4(\mathrm{~s}, 3 \mathrm{H}, \mathrm{CH} 3), 4.9$ (d, J = 4.7Hz, 1H, ArH), 6.1 $(\mathrm{d}, \mathrm{J}=4.7 \mathrm{~Hz}, 1 \mathrm{H}, \mathrm{ArH}), 7.3(\mathrm{~m}, 6 \mathrm{H}, \mathrm{ArH}), 7.9(\mathrm{~d}, \mathrm{~J}=6.8 \mathrm{~Hz}, 2 \mathrm{H}, \mathrm{ArH}) \mathrm{ppm} ;{ }^{13} \mathrm{C} \mathrm{NMR}\left(\mathrm{CDCl}_{3}\right)$,

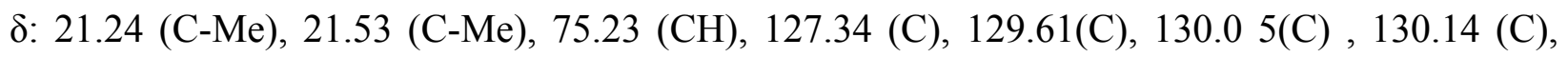
$131.52(\mathrm{C}), 137.50(\mathrm{C}), 138.40(\mathrm{C}), 144.11(\mathrm{C}), 198.24(\mathrm{C}=\mathrm{O})$ ppm; MS: $\left(\mathrm{M}^{+}\right)$: $(\mathrm{EI})$ : exact mass calcd for $\mathrm{C}_{16} \mathrm{H}_{16} \mathrm{O}_{2}, 240.1150$; found 240.1150, 240, 239, 238, 224, 119, 120, 122, 107, 92, 77, 51. 
Reduction of 1-(4-methoxyphenyl)-2-phenylethane-1,2-dione (3a) by baker's yeast in EtOAc : (-)- 2-hydroxy-1-(4-methoxyphenyl)-2-phenylethanone, ((-)-4a $\left.\mathrm{C}_{15} \mathrm{H}_{14} \mathrm{O}_{3}\right)$

A similar procedure as used for (1a) was applied. The elution solvent was 1:3 (v:v) Et $\mathrm{H}_{2} \mathrm{O}$ :ligroin to afford $0.38 \mathrm{~g}, 50.7 \%$ of (-)-4a, mp $106{ }^{\circ} \mathrm{C}$, (acetone, $\mathrm{c}=0.042 \mathrm{M}$, [a $]_{\mathrm{D}}{ }^{25}=-84$; IR (KBr): 3460 $(\mathrm{OH}), 3070$ ( $\mathrm{CH}$ stretching), 2920 ( $\mathrm{CH}$ stretching), 2830 ( $\mathrm{CH}$ stretching), $1660(\mathrm{C}=\mathrm{O}), 1600$ $(\mathrm{C}=\mathrm{C}), 1565(\mathrm{C}=\mathrm{C}), 1500,1260,1175,1070,1060,975,820,740,700 \mathrm{~cm}^{-1}$; ${ }^{1} \mathrm{H}$ NMR $\left.\left(\mathrm{CD}_{3} \mathrm{COCD}_{3}\right), \delta: 3.7\left(\mathrm{~s}, 3 \mathrm{H}, \mathrm{CH}_{3}\right), 4.5(\mathrm{~d}, \mathrm{~J}=4.7 \mathrm{~Hz}, 1 \mathrm{H}, \mathrm{OH})\right), 5.8(\mathrm{~d}, \mathrm{~J}=4.7 \mathrm{~Hz}, 1 \mathrm{H}, \mathrm{CH}), 6.8$ $(\mathrm{d}, \mathrm{J}=6.8 \mathrm{~Hz}, 2 \mathrm{H}, \mathrm{ArH}), 7.2(\mathrm{~m}, 5 \mathrm{H}, \mathrm{ArH}), 7.8(\mathrm{~d}, \mathrm{~J}=6.8 \mathrm{~Hz}, 2 \mathrm{H}, \mathrm{ArH}) \mathrm{ppm} ;{ }^{13} \mathrm{C} \mathrm{NMR}$ $\left(\mathrm{CD}_{3} \mathrm{COCD}_{3}\right), \delta: 54.58(\mathrm{C}-\mathrm{Me}), 75.16(\mathrm{CH}), 113.32(\mathrm{C}), 126.49(\mathrm{C}), 127.02(\mathrm{C}), 127.38(\mathrm{C})$, 128.14 (C), 130.93 (C), $140.06(\mathrm{C}), 163.4(\mathrm{C}), 196.96(\mathrm{C}=\mathrm{O})$ ppm. $\lambda_{\max }=212,280 \mathrm{~nm}$; MS: $\left(\mathrm{M}^{+}\right)$: (EI): exact mass calcd for $\mathrm{C}_{15} \mathrm{H}_{14} \mathrm{O}_{3}, 242.0943$; found 242.0942, 242, 240, 241, 227, 226, $224,207,135(100 \%), 127,121,107,92,77,64,51$. The spectroscopic data of $\left((+)-4 \mathrm{a} \mathrm{C}_{15} \mathrm{H}_{14} \mathrm{O}_{3}\right)$ has been reported. ${ }^{5 \mathrm{a}}$

Reduction of 1-(4-(dimethylamino)phenyl)-2-phenylethane-1,2-dione (3b) in EtOAc by baker's yeast : (+)- 1-(4-(dimethylamino)phenyl)-2-phenylethanone, (+)-4b $\left.\mathrm{C}_{16} \mathrm{H}_{17} \mathrm{NO}_{2}\right)$

A similar procedure as used for (1a) was applied. The elution solvent was 1:3 (v:v) $\mathrm{Et}_{2} \mathrm{O}$ :ligroin to afford $0.18 \mathrm{~g}, 51.4 \%$ of $(+)-4 \mathrm{~b}, \mathrm{mp} 162{ }^{\circ} \mathrm{C}$, (acetone, $\mathrm{c}=0.019 \mathrm{M}$, [a] $]_{\mathrm{D}}^{25}=-56.4^{\mathrm{o}} ; \mathrm{IR}(\mathrm{KBr})$ : $3400(\mathrm{OH}), 3070(\mathrm{CH}$ stretching), $2910(\mathrm{CH}$ stretching), $2800,1650(\mathrm{C}=\mathrm{O}), 1605(\mathrm{C}=\mathrm{C})$, 1286, 1540, 1440, 1385, 1165, 1090, 1065, 975, 800, 750, $700 \mathrm{~cm}^{-1} ;{ }^{1} \mathrm{H} \mathrm{NMR}\left(\mathrm{CDCl}_{3}\right) \delta: 2.9(\mathrm{~s}$, $6 \mathrm{H}, 2 \mathrm{xMe}), 4.8(\mathrm{~d}, \mathrm{~J}=6.1 \mathrm{~Hz}, 1 \mathrm{H}, \mathrm{OH}), 5.8(\mathrm{~d}, \mathrm{~J}=6.1 \mathrm{~Hz}, 1 \mathrm{H}, \mathrm{CH}), 6.5(\mathrm{~d}, \mathrm{~J}=9 \mathrm{~Hz}, 2 \mathrm{H}, \operatorname{ArH})$,

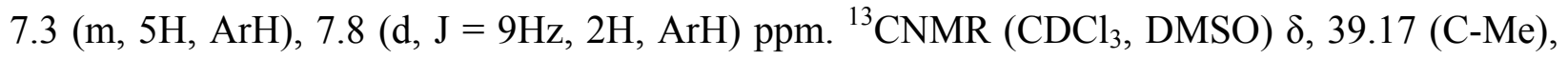
$75.42(\mathrm{CH}), 110.76(\mathrm{C}), 120.54(\mathrm{C}), 127.12$ (C), 127.30 (C), 128.64 (C), 131.76 (C), 140.50 (C), $153.43(\mathrm{C}), 196.32(\mathrm{C}=\mathrm{O}), \lambda_{\max }=212,240$ and $353 \mathrm{~nm}$. ; MS: $\left(\mathrm{M}^{+}\right)$: (EI): exact mass calcd for $\mathrm{C}_{16} \mathrm{H}_{17} \mathrm{NO}_{2}, 255.1259$; found 255.1262, 255, 251, 222, 207 (100\%), 205, 177, 165, 145, 105, 91, $67,57$.

\section{Acknowledgements}

The financial support of the Research Committee of Guilan University Fund is gratefully acknowledged. We also acknowledge the useful suggestions made by Professor John L. Belletire of Adelphia Pharma, USA.

\section{References and Notes}

1. (a) Faber, K. Biotransformations in Organic Chemistry, 4th Edn.; Springer: New York, 2000; (b) Liese, A.; Seebach, K.; Wandrey, C. Industrial Biotransformations; Wiley-VCH: Weinheim, 2000.

2. Chin-Joe, I.; Nelisse, P. M.; Straathof, A. J. J.; Jongejan, J. A.; Pronk, J. T.; Heijnen, J. J. Biotechnol Bioeng. 2000, 69, 370. 
3. (a) Koike, T.; Murata, K.; Ikariya, T.; Org. Lett. 2000, 2, 3833; (b) Coppola, G. M.; Schuster, H. F. $\alpha$-Hydroxy Acids in Enantioselective Synthesis; VCH: Weinheim, 1997; (c) Davies, F. A.; Chen, B. C. Chem. Rev. 1992, 92, 919; (d) Hashiyama, T.; Morikawa, K.; Sharpless, K. B. J Org. Chem. 1992, 57, 5067; (e) Knight, R. L.; Leeper, F. G. J. J. Chem. Soc., Perkin Trans 1 1998, 1891; (f) Enders, D. Breuer, K.; Teles, J. H. Helv. Chim. Acta. 1996, 79, 1217; (g) Enders, D.; Breuer, K.; Comprehensive Asymmetric Catalysis, Jacobsen, E. N.; Pfaltz, A.: Yamamoto, H. Edn., Springer: Berlin, 1999; Vol. 2, p 1093; (h) Lee, J. C.; Jin, Y. S.; Choi, J. H. Chem. Commun. 2001, 956; (i) Forni, A.; Caselli, E.; Prati, F.; Bucciarelli, M.; Torre, G. Arkivoc 2002, 12, 123

4. (a) Hanessian, S. Total Synthesis of Natural Products: The Chiron Approach. Pergamon: New York, 1983, Chap. 2. (b) Maguire, A. R.; Collins, S. G.; Ford, A. Arkivoc 2003, 7, 96.

5. (a) Mahmoodi, N. O.; Mohamadi, H.G. Monatsh. Fur. Chem. 2003, 134, 1283; (b) Demir, A. S.; Hamamci, H.; Ayhan, P.; Duygu, A. N.; Cigdem -Igdira, A.; Capanoglub, D. Tetrahedron: Asymmetry 2004, 15, 2579; (c) Maruyama, R.; Nishizawa, M.; Itoi, Y.; Ito, S.; Inoue, M. Journal of Biotechnology 2002, 94, 157; (d) Qun, J.; Shanjing, Y.; Lehe, M. Enzyme and Microbial Technology 2002, 30, 721; (e) Mahmoodi, N.O.; YousefiMalekroudi, R. Russ. J. Org. Chem. 2006, 3, 365; (f) Mahmoodi, N.O.; Tajik, H.; Tabatabaeian, K.; Shahbazi, M. J. Serb. Chem. Soc. The enantioselective $\beta$-ketoester reductions by Saccharomyces cerevisiae will be appear in 2006, volume 71 content; http://www.shd.org.yu/HtDocs/SHD/vol71/ Contents.html; (g) Buisson, D.; El Baba, S.; Azerad, R. Tetrahedron Lett. 1986, 27, 4453.

6. Chu, Y.; Li, B, Silvestre, V. Z.; Cheng, J. P. Bioorganic Chemistry 2006, 34, 158; (b) Mihovilovic, M. D.; Spina, M.; Stanetty, P. Arkivoc 2005, (v), 33.

7. Conceio, G. J. A.; Moran, P. J. S.; Rodrigues, J. A. R. Arkivoc 2003, 10, 500.

8. Jayasinghe, L.Y., Kodituwakku, D., Smallridge, A. J., Trewhella, M. A., Bull. Chem. Soc. Jpn. 1994, 67, 2528.

9. Kramer, A. Pfader, H. Helv. Chim. Acta 1982, 65, 293.

10. Dumanski, P.G. Journal of Molecular Catalysis B: Enzymatic 2001, 11, 905.

11. Sybesma, H. W. F.; Straathof, A. A. J.; Jongejan, J. A.; Pronk, J. T.; Heijnen, J. J. Biocatal. Biotransform. 1998, 16, 95.

12. Medson, C.; Smallridge, A. J.; Trewhella, M. A.; Tetrahedron: Asymmetry 1997, 8, 1049.

13. Mahmoodi, N. O.; Emadi, S. Russ. J. Org. Chem. 2004, 40, 377.

14. (a) Adam, W.; Diaz, M. T.; Feel, R. T.; Saha-Moller, C. R. Tetrahedron: Asymmetry 1996, 8, 2207; (b) Adam, W.; Feel, R. T.; Saha-Moller, C. R.; Zhao, C. G. Tetrahedron: Asymmetry 1998, 9, 397; (c) Gala, D.; DiBenedetto, D. J.; Clark, J. E.; Murphy, B. L.; Schumacher, D. P.; Steinman, M. Tetrahedron Lett. 1996, 37, 611; (d) Kajiro, H.; Mitamura, S.; Mori, A.; Hiyama, T. Tetrahedron: Asymmetry 1998, 9, 907.

15. (a) Enders, D.; Breuer, K.; Teles, J. H. Helv Chim Acta 1996, 79, 1217; (b) Dictionaryof Organic Compounds, Pennsylvania: Chapman and Hall: 1982, 5th Edn.; (c) Gu, J. X.; Li, Z. 
Y.; Lin, Q., J. Chin. Lett. 1995, 457; (d) Catalogue. Handbook of Fine Chemicals, Aldrich Chemical Company, Ltd., England, 1989.

16. (a) Vogel, A.I. Textbook of Practical Organic Chemistry: 1980, 4th Edn. William Clows Limited.

17. (a) Ide, W. S., Buck, J. S. Org. React. 1948, 4, 269; (b) Kuebrich, J. P.; Schowen, R. L.; Wang, M.; Lupes, M. E. 1971, 93, 121.

18. Demir, A. S.; Dunnwald, T.; Iding; H.; Pohl, M.; Muller, M., Tetrahedron: Asymmetry 1999, 10, 4769. 